\title{
Very high resolution optical transition radiation imaging system: Comparison between simulation and experiment
}

B. Bolzon, ${ }^{1,2,3,{ }^{*}}$ A. Aryshev, ${ }^{4}$ T. Aumeyr, ${ }^{5}$ S. Boogert, ${ }^{5}$ P. Karataev, ${ }^{5}$ K. O. Kruchinin, ${ }^{5}$ T. Lefevre, ${ }^{1}$ S. Mazzoni, ${ }^{1}$ L. Nevay, ${ }^{5}$ M. Shevelev, ${ }^{4}$ N. Terunuma, ${ }^{4}$

J. Urakawa, ${ }^{4}$ and C. P. Welsch ${ }^{2,3}$

${ }^{1}$ CERN, CH-1211 Geneva 23, Switzerland

${ }^{2}$ The University of Liverpool, Liverpool L69 3BX, United Kingdom

${ }^{3}$ Cockcroft Institute, Daresbury, Warrington, Cheshire WA4 4AD, United Kingdom

${ }^{4}$ KEK: High Energy Accelerator Research Organization, 305-0801 Tsukuba, Ibaraki, Japan

${ }^{5}$ John Adams Institute at Royal Holloway, University of London, Egham, Surrey TW20 OEX, United Kingdom

(Received 10 September 2014; revised manuscript received 15 May 2015; published 31 August 2015)

Optical transition radiation (OTR) has become a commonly used method for 2D beam imaging measurements. In the Accelerator Test Facility 2 (ATF2) at KEK, beam sizes smaller than the OTR point spread function have been measured. Simulations of the OTR imaging system have been performed using the ZEMAX software to study the effects of optical errors such as aberrations, diffraction, and misalignments of optical components. This paper presents a comparison of simulations of the OTR point spread function with experimental data obtained at ATF2. It shows how the quantification and control of optical errors impacts on optimizing the resolution of the system. We also show that the OTR point spread function needs to be predicted accurately to optimize any optical system and to predict the error made on measurement.

DOI: 10.1103/PhysRevSTAB.18.082803

PACS numbers: 41.60.Dk, 41.85.Qg, 42.15.Eq, 29.27.Fh

\section{INTRODUCTION}

In accelerators, optical transition radiation (OTR) beam imaging systems are widely used to measure the transverse properties of a particle beam. The resolution of OTR monitors is normally defined as the root mean square of the so-called OTR point spread function (PSF) [1]. It corresponds to the projection by an optical system of the OTR field distribution generated by a single particle. It contains information about both the actual source distribution and the imperfections of the optical system.

There have been several theoretical models developed [2-5] to define an OTR source distribution and study the resolution of OTR imaging systems. They are typically based on analytical calculations propagating the OTR electric field using diffraction laws through ideal lenses, i.e., based on thin lens approximation. Optical errors, such as spherical and chromatic aberrations, as well as misalignments of optical components including the transverse displacement or the tilt of a lens, could not be calculated accurately. Hence the resolution of the OTR imaging system can only be estimated with a large uncertainty.

\footnotetext{
*Benoit.Bolzon@cern.ch

Published by the American Physical Society under the terms of the Creative Commons Attribution 3.0 License. Further distribution of this work must maintain attribution to the author(s) and the published article's title, journal citation, and DOI.
}

In order to study these limitations, numerical simulations with the ZEMAX optical design program [6] have been performed. This code offers a large database of commercially available optical components and integrates all the features required to design, optimize, and tolerate virtually any optical system.

Using the ultralow emittance beam available at the Accelerator Test Facility at KEK in Japan, the OTR PSF has been observed [7] and a method to measure beam sizes smaller than the transverse width of the PSF has been established by measuring the visibility of the PSF [8].

This paper presents simulations of the propagation of the OTR electric field in both near- and far-field conditions. The point spread function has been studied as a function of the observation wavelengths and the properties of the lenses. The simulated resolution of the OTR imaging system used in ATF2 is then discussed and compared to experimental data.

\section{SIMULATIONS USING ZEMAX}

ZEMAX is widely used in the optics industry as a standard design tool. OTR PSF simulations are performed using the physical optics propagation (POP) mode, which, using diffraction laws, propagates a wave front through an optical system surface by surface. The wave front is modeled at every surface using an array of discretely sampled points, each of them storing complex amplitude 
information about the photon beam. The entire array is then propagated in free space between optical surfaces. At each optical surface, a transfer function is computed which propagates the beam from one side of the optical surface to the other. To propagate the field from one surface to the other, either Fresnel diffraction propagation or an angular spectrum propagation algorithm is used. ZEMAX automatically chooses the algorithm that yields the highest numerical accuracy. In POP mode, the entire beam array must be stored in computer memory at once, which may result in a large quantity of RAM needed to accurately model the propagation of a transversely large photon source.

Any source of light can be provided in POP mode. The user has to define the spatial distribution of the complex electric field of the source either in a beam file or in a Windows dynamic link library (DLL). We input to ZEMAX the approximation of the electric field for the OTR vertical polarization component induced by a single electron on a target surface [4], defined as follows:

$$
\begin{aligned}
& \operatorname{Re}\left(E_{y}\right)=\operatorname{const} \frac{Y}{\sqrt{X^{2}+Y^{2}}}\left[\frac{2 \pi}{\gamma \lambda} K_{1}\left(\frac{2 \pi}{\gamma \lambda} \sqrt{X^{2}+Y^{2}}\right)-\frac{J_{0}\left(\frac{2 \pi}{\gamma \lambda} \sqrt{X^{2}+Y^{2}}\right)}{\sqrt{X^{2}+Y^{2}}}\right] \\
& \operatorname{Im}\left(E_{y}\right)=0
\end{aligned}
$$

with $X$ and $Y$ the two orthogonal coordinates of the target surface measured from the point of electron incidence, $\gamma$ is the relativistic Lorentz factor, $\lambda$ is the radiation wavelength, $K_{1}$ is the modified Bessel function of first order, and $J_{0}$ is the Bessel function of zeroth order.

An example of the spatial distribution of the vertical polarization component of the OTR irradiance at the target surface generated by a single particle is depicted in Fig. 1.

\section{A. Free space propagation}

As mentioned previously, the size of the array used in ZEMAX to define the OTR field at the source is crucial for providing accurate predictions. To quantify this effect, several simulations have been performed for different transverse sizes of the OTR field distribution at the source. The resulting OTR field is then propagated in free space over a distance long enough to reach far-field conditions,

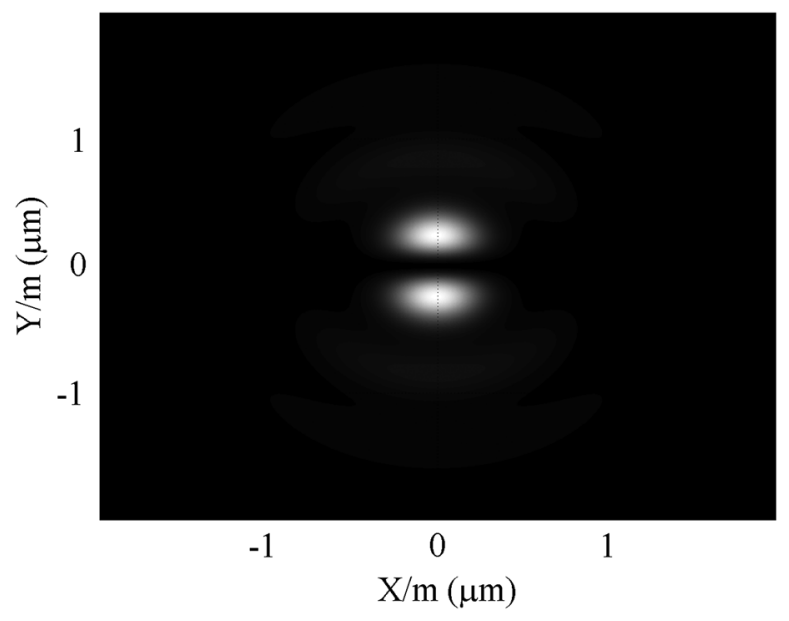

FIG. 1. Simulation of the 2D distribution of vertically polarized OTR irradiance generated at the target by a single electron. with the observation plane distant from the source by a distance $L \gg \lambda \gamma^{2} /(2 \pi)$ [9]. The corresponding OTR angular distribution can thus be compared to analytical models [2] that predict the well-known OTR double lobes angular distribution peaking at $1 / \gamma$. The results are displayed in Fig. 2, where the transverse size of the OTR field is expressed as a radius normalized to $\gamma \lambda$. For small radii, the truncation of the OTR field generates an artificial diffraction pattern that results in a wider irradiance distribution in the far-field condition. It clearly indicates that the simulation of the OTR tails is essential up to a distance significantly larger than $\lambda \gamma /(2 \pi)$, which satisfy the infinite target dimension criterion, that most analytical models assume.

Despite the required computing time and resource increase, all simulations performed in this paper assume a radius of the transverse EM field at the source equal to 4 times $\lambda \gamma$ and a $65000 \times 65000$ matrix size. Figure 3 shows the OTR angular distribution simulated by ZEMAX for a

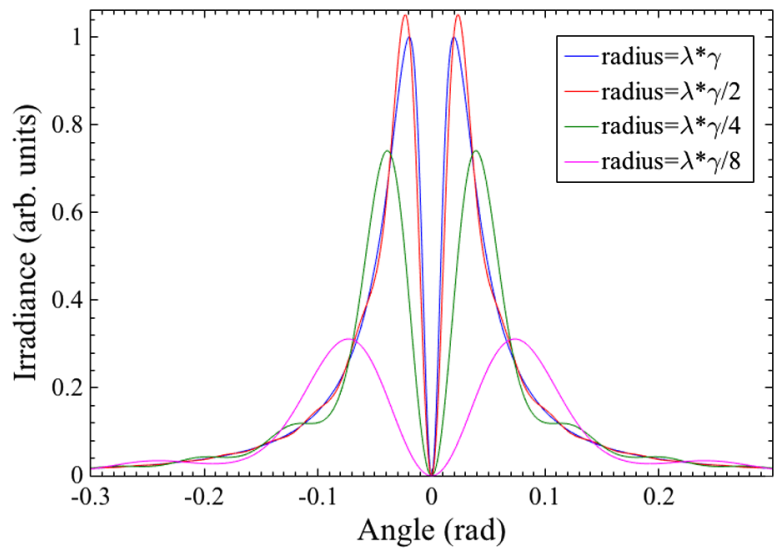

FIG. 2. The far-field OTR angular distribution calculated by ZEMAX for different OTR field radius $(\lambda=550 \mathrm{~nm} ; \gamma=50)$. 


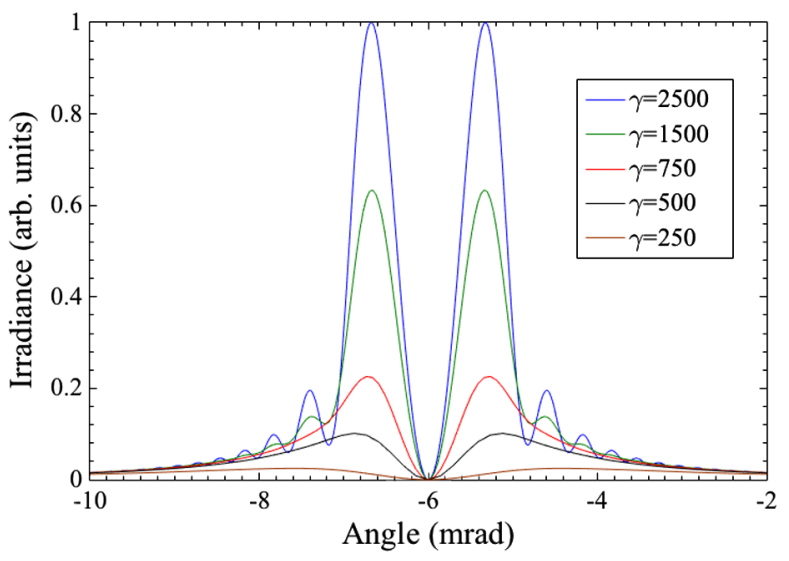

FIG. 3. OTR angular distribution calculated by ZEMAX for $\lambda=550 \mathrm{~nm}$ and for different energies.

wavelength of $550 \mathrm{~nm}$ and for different energies. The OTR angular distribution in the far field has the typical double lobe structure as expected from analytical models [2]. The peak intensity is proportional to $\gamma^{2}$. More results on OTR simulations using ZEMAX in a far field can be found in Ref. [10].

One should note that depending on the beam energy, the optical elements of an imaging system, located close to the source, are either in the prewave zone [5] or the near-field region, assuming distances $L$ from the radiation source satisfying the condition $\lambda \gamma / 2 \pi \ll L \leq \lambda \gamma^{2} / 2 \pi$ or $<\lambda \gamma / 2 \pi$, respectively. The transverse distribution of the OTR field at this specific position cannot be calculated anymore assuming a far-field condition for which OTR light is described by its angular distribution as presented in this paragraph.

\section{B. Point spread function}

The OTR PSF is the projection by an optical system of the OTR field distribution generated by a single particle. For all ZEMAX simulations presented in the following paragraph, we consider the case of the ATF2 OTR imaging system described in Sec. III A. In Fig. 4, the vertical line profiles of the simulated OTR PSF are presented for several beam energies. The vertical dimension $Y / m$ is normalized to the optical magnification so that it represents the dimension of the field at the position of the source.

The spatial distribution has a minimum in the center and two main peaks separated by a distance which varies very little with beam energy, as expected from theoretical models [5]. It only changes from $6.0 \mu \mathrm{m}$ for $\gamma=50$ to $7.1 \mu \mathrm{m}$ for $\gamma=2500$. In Fig. 5 the OTR PSF of the same optical system has been simulated for different wavelengths. The simulations have been performed for a beam energy corresponding to the experimental beam conditions at ATF2 $(\gamma=2500)$. The position of the observation plane was adjusted accordingly for each wavelength. As expected, diffraction at the source is smaller for shorter

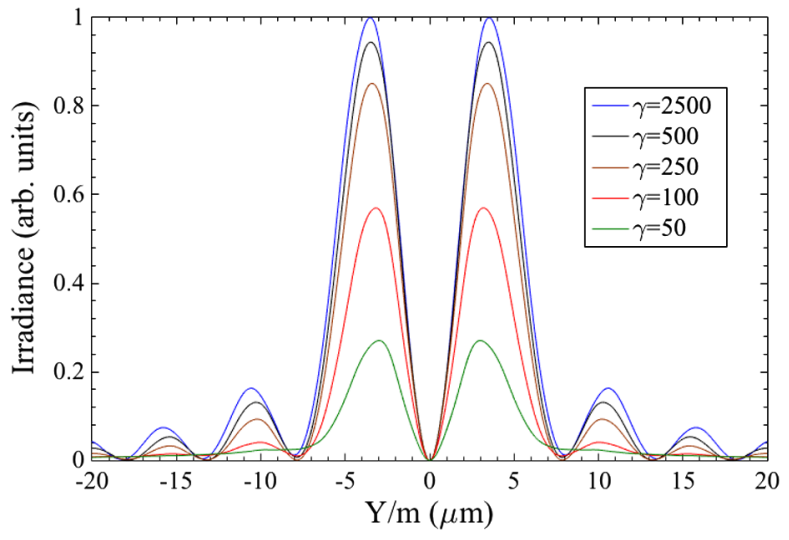

FIG. 4. OTR PSF distribution simulated by ZEMAX for $\lambda=550 \mathrm{~nm}$ and for different energies.

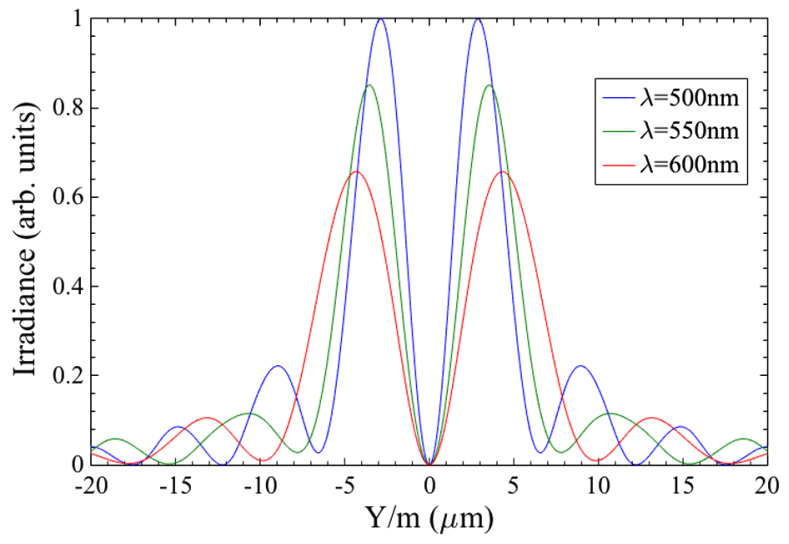

FIG. 5. OTR PSF distribution simulated by ZEMAX for $\gamma=2500$.

wavelengths, and the size of the PSF reduces for shorter wavelengths.

\section{Optimization of the lens}

The choice of the lens is crucial for an optical system. Simulations have been performed for three different lenses with the same diameter of $30 \mathrm{~mm}$ and focal length of $100 \mathrm{~mm}$, providing the same optical magnification of 7.4. The plano-convex lens, SLB-30-100-PY2 from SigmaKoki, that was used in the first version of the OTR imaging system installed on ATF2 [8] has been taken as a reference for comparison. Simulations using either a visible achromatic doublet lens, DLB-30-100-PM from Sigma-Koki, or an UV achromatic doublet lens 027-3020 from OptoSigma, have been performed and the results are presented in Fig. 6 .

The visible achromatic lens gives a smaller PSF than the plano-convex lens because chromatic and spherical aberrations are better corrected. However, it also provides a smaller PSF than the UV achromatic lens. Even if using a shorter wavelength was expected to reduce diffraction effects, UV materials induce larger aberrations, which 


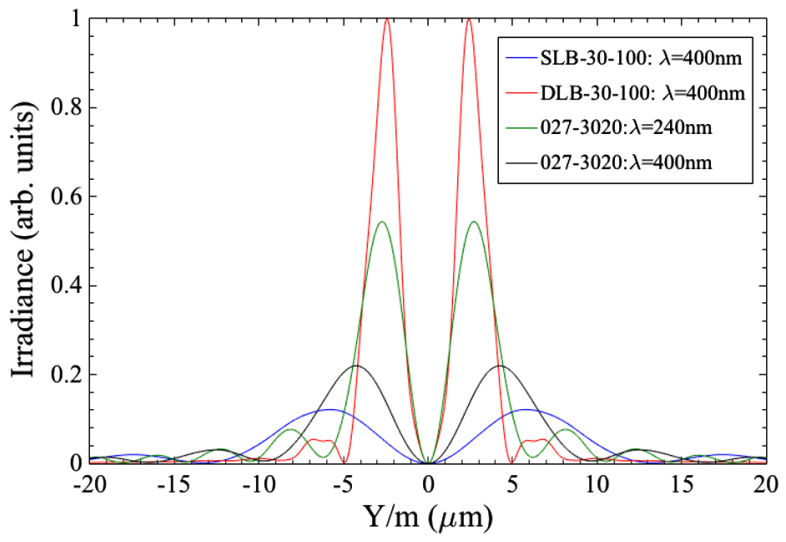

FIG. 6. OTR PSF distribution calculated by ZEMAX for different types of lenses and for $\gamma=2500$.

clearly limit the use of such lenses. An alternative solution would be to work in the UV domain with reflective optics, i.e., elliptical mirrors.

\section{Measuring beam size with OTR}

In order to predict the ability of an OTR imaging system to measure the beam size, a series of simulations have been performed assuming Gaussian beam sizes with $\sigma$ values ranging from $750 \mathrm{~nm}$ to $20 \mu \mathrm{m}$. The expected beam profiles are obtained by convoluting these Gaussian profiles with the OTR PSF of the imaging system presented previously. The results are shown on Fig. 7. The amplitude of all profiles is normalized to one for clarity and the PSF is also presented for comparison.

Beam sizes smaller than $3 \mu \mathrm{m}$ sigma can be measured using the visibility of the PSF [8]. The transverse distribution of the OTR point spread function as described in more detail in Sec. II B is null in the center and has two main lobes at a distance that varies depending on the observation wavelength and the characteristic of the optical system in use. As shown in Fig. 7, for increasing beam sizes, the central part of the distribution gets larger and

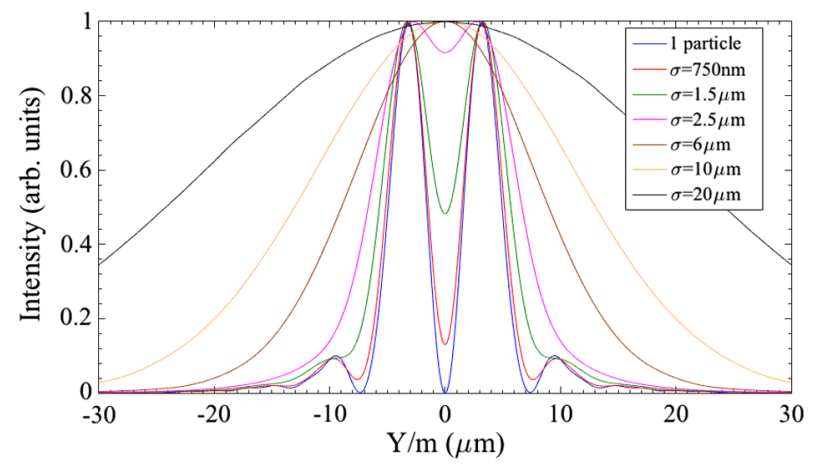

FIG. 7. Expected vertical beam profile obtained from the convolution of the ATF2 OTR PSF with a Gaussian beam distribution. Simulations are performed for $\lambda=550 \mathrm{~nm}$ and $\gamma=2500$.

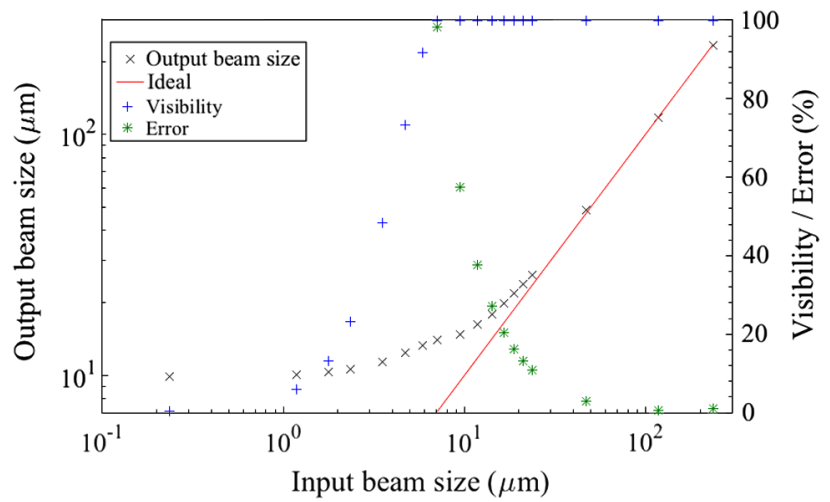

FIG. 8. Simulated output beam size versus input beam size. The line in red shows the ideal curve where output beam size equals the input beam size.

larger. The beam size is then proportional to the so called "visibility" of the OTR PSF, which can be defined as the ratio $I_{\min } / I_{\max }$, where $I_{\min }$ is the amplitude of the distribution at the center (i.e., zero for the PSF) and $I_{\max }$ the maximum amplitude of the distribution (i.e., corresponding to the main lobe). The corresponding visibility $I_{\min } / I_{\max }$ is plotted in Fig. 8 and will allow the measurement of beam sizes with $\sigma$ (FWHM) values ranging from $0.72(1.7)$ up to $2.5(6) \mu \mathrm{m}$ with visibilities of 0.13 and 0.92 , respectively. Measuring smaller beam sizes will then depend on the capability of measuring a precisely very small value of $I_{\min }$, which will be limited experimentally by signal-tonoise issues.

There is a range (blind area) of beam sizes for which the visibility of the PSF has disappeared but the transverse size of the measured distribution is still dominated by the width of the PSF. In such conditions, a Gaussian fit cannot be applied anymore and in Fig. 8, the beam sizes are then displayed using FWHM values. It is thus shown that for a $7 \mu \mathrm{m}$ FWHM ( $3 \mu \mathrm{m} \sigma)$ input beam size, the error made on the measurement is in excess of $98 \%$, if the PSF contribution is not subtracted. This error diminishes rapidly for larger beam sizes. It reduces to $3 \%$ for a beam size of $45 \mu \mathrm{m}$ FWHM, the error becoming then negligible for even larger beam sizes. However, the blind area boundaries depend on the wavelength of light and longitudinal position of the focusing length. By changing either of them one may cover the blind area and achieve a continuous beam size diagnostics range.

\section{EXPERIMENTAL VALIDATION}

\section{A. Experimental setup at ATF2}

ATF2 [11] is the linear collider final-focus test beam line that aims to focus the low-emittance beam from the ATF damping ring to a vertical beam size of about $37 \mathrm{~nm}$ and at the same time to demonstrate nanometer pointing stability, using numerous advanced beam diagnostics and feedback 
TABLE I. ATF2 beam parameters.

\begin{tabular}{lc}
\hline \hline Energy $(\mathrm{GeV})$ & 1.28 \\
V/H emittance (m.rad) & $2.5 / 200 \times 10^{-11}$ \\
Bunch population & $10^{10}$ \\
\hline \hline
\end{tabular}

tools [12]. The facility's main beam parameters are summarized in Table I.

The beam size in the vertical plane can reach submicrometer dimensions, which is equivalent or smaller than the size of the OTR point spread function. The imaging system, as presented in Fig. 9, was designed to study the OTR PSF [7] and measure very small beam sizes using the PSF visibility technique. This optical transition radiation screen was part of the laser wire scanner system developed on ATF2 over the past ten years to measure noninvasively sub- $\mu \mathrm{m}$ transverse beam profiles [13].

The optical system, optimized in 2013, is based on an achromatic doublet lens, DLB-30-120-PM, from SigmaKoki, providing a magnification of 7.4 [8]. It is composed of four aluminum mirrors forming a periscope, an optical polarizer, and a set of chromatic band-pass filters. The OTR screen is an aluminum coated silicon wafer. The camera, model SBIG-ST8300M, is based on a Kodak KAF-8300 (monochrome) sensor with $5.4 \mu \mathrm{m}$ pixel size, $3352 \times 2532$ pixel array. It offers $50 \%$ quantum efficiency and a lownoise read-out electronic with 16 bit dynamic range. A remotely controlled iris inserted one centimeter upstream of the lens allows studying spherical aberration and diffraction effects. One should note that the lens of the optical system is located in the so called prewave zone.

A typical image with the corresponding vertical and horizontal beam profiles is depicted in Fig. 10 already

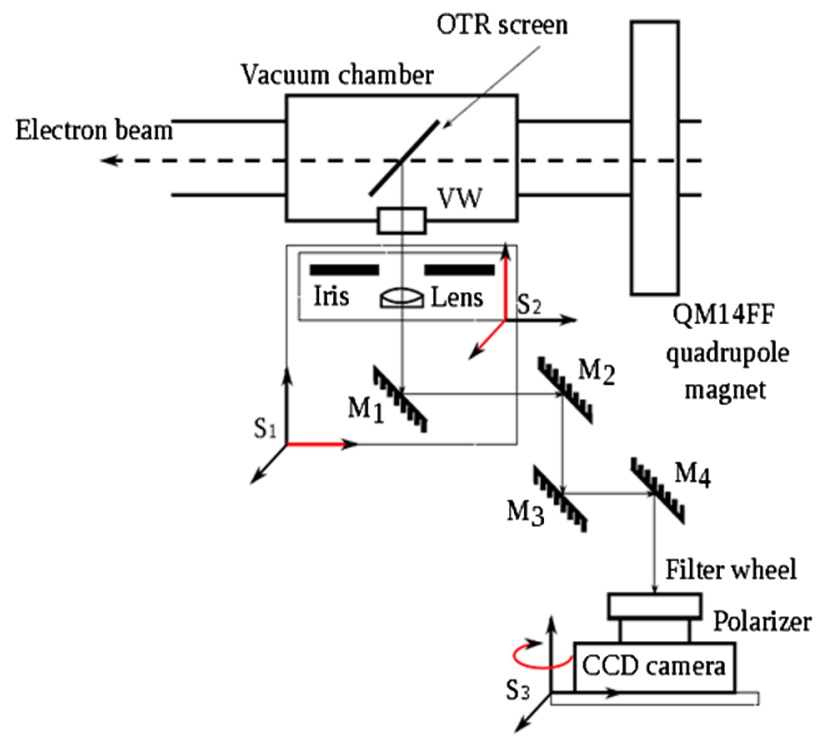

FIG. 9. Experimental setup of the high-resolution OTR imaging system at ATF2/KEK. presented in [8]. With a large optical magnification, the depth of field of the optical system is small compared to the typical horizontal beam size. Since the OTR screen is tilted horizontally at $45^{\circ}$ with respect to the beam trajectory, the observed images show a large smearing along the horizontal plane; see Fig. 10(a). However this effect does not affect the light intensity, and horizontal beam profiles are simply measured projecting the image on the horizontal plane and fitting the corresponding curve by a Gaussian distribution. It is measured in this particular case to $52 \mu \mathrm{m}$.

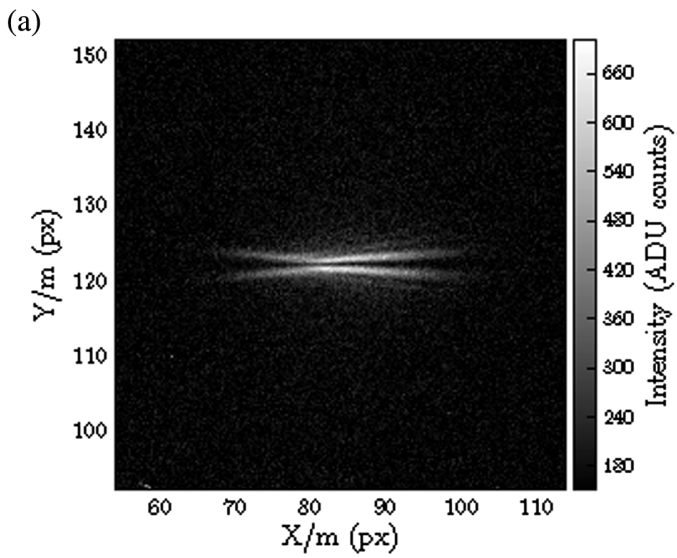

(b)

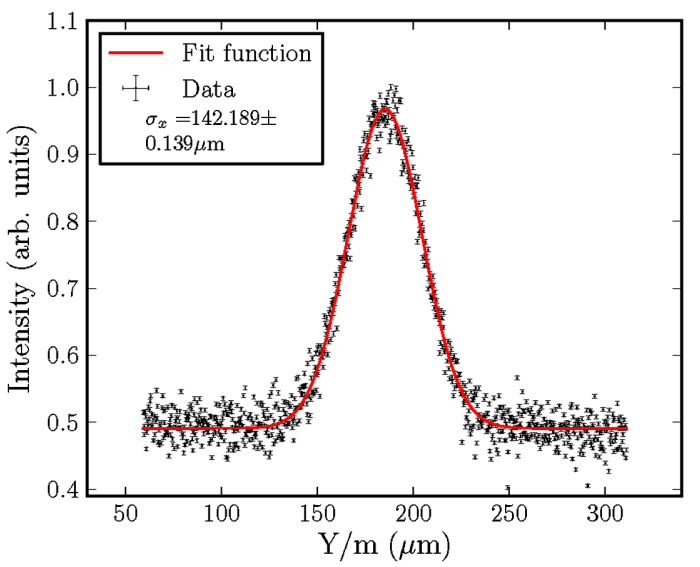

(c)

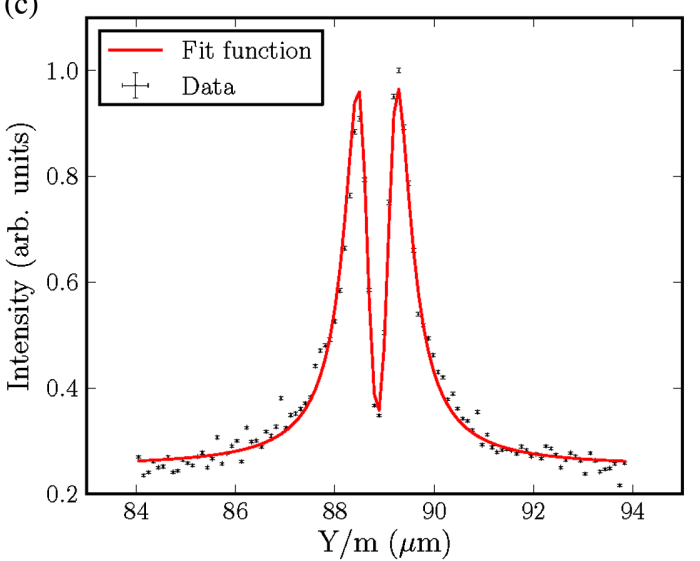

FIG. 10. Typical beam image (a) and corresponding horizontal (b) and vertical (c) profiles. 
Because of this blurring effect, the vertical beam profile is extracted selecting only a small region in the central part of the image and projecting it on the vertical plane. An empirically found fit function had been introduced to analyze the corresponding profile:

$f(x)=a+\frac{b}{1+\left[c\left(x-x_{0}\right)\right]^{4}}\left\{1-e^{-2 c^{2} \sigma^{2}} \cos [c(x-\Delta x)]\right\}$.

$a, b, c, \sigma$, and $\Delta x$ are free parameters of the fit function, namely, $a$ is the vertical offset of the distribution with respect to zero which included a constant background; $b$ is the amplitude of the distribution; $c$ is the distribution width; $\sigma$ is the smoothing parameter dominantly defined by the beam size; and $\Delta x$ is the horizontal offset of the distribution with respect to zero. From the fitted curve as seen in Fig. 10(c), the visibility of the point spread function is retrieved and a vertical beam size of $750 \mathrm{~nm}$ has been evaluated in this case.

In [8] we demonstrated that, for flat transverse beam distributions with vertical beam size in submicrometer range, both beam sizes are retrieved using only the vertically polarized OTR photons.

Moreover, at the location of the OTR screen, the ATF2 beam optic can be tuned so that the vertical beam size reaches a value small enough so that the beam size contribution cannot be measured anymore. The resulting images provide thus direct measurements of OTR PSF. The following paragraphs present a comparison between the PSF measured experimentally and the ones simulated by ZEMAX.

\section{B. Tuning of the image plane}

On the optical table, the distances between the OTR target and the lens, as well as between the lens and the CCD camera, are only known within millimeter accuracy. In order to find the image plane, the lens is installed on a translation stage and moved longitudinally by steps of $100 \mu \mathrm{m}$ until the measured width of the PSF reaches a minimum. The evolution of the distance between the PSF main lobes is shown in Fig. 11 as a function of the lens position for a wavelength of $550 \mathrm{~nm}$. The lens was moved in both directions. The distance between lobes increases by a factor 5 for offset position of the lens of $500 \mu \mathrm{m}$. Simulated and experimental data show an excellent agreement for positive offset, i.e., the distance between the lens and the camera increases. The data corresponding to negative offset are not depicted in the plot because even if the OTR distribution becomes wider, which would indicate that we move away from the image plane, the two-lobes distribution disappears and makes the comparison with the case of positive offsets impossible.

The PSFs measured experimentally for three different wavelengths are presented in Fig. 12. The longitudinal position of the lens has to be adjusted for each wavelength.

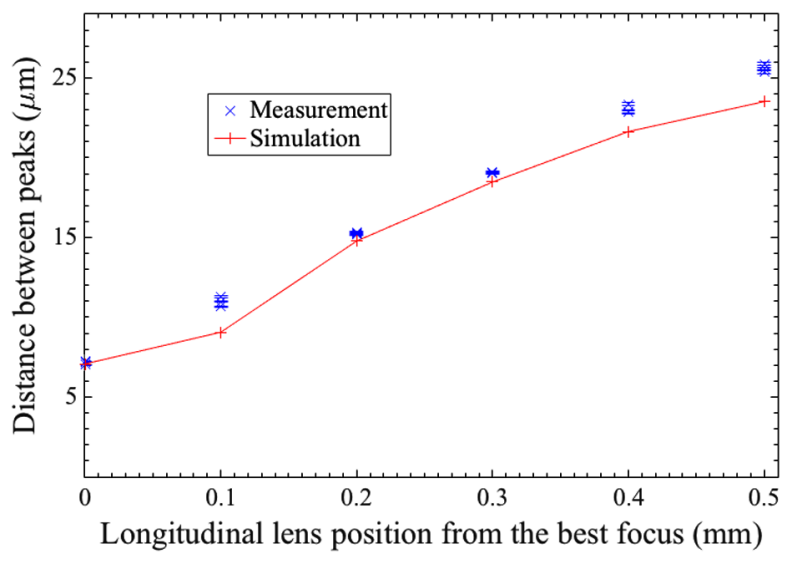

FIG. 11. Distance between OTR PSF peaks as a function of the longitudinal position of the lens $(\gamma=2500 ; \lambda=550 \mathrm{~nm})$.

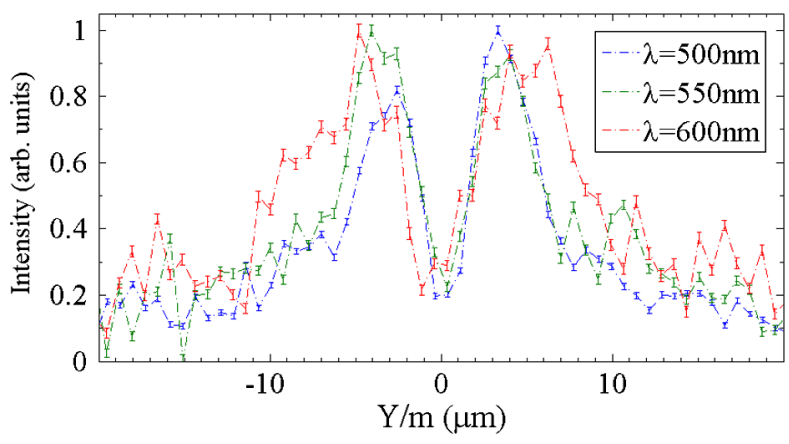

FIG. 12. OTR PSF measured at best focus for 500, 550, and $600 \mathrm{~nm}$ wavelength optical filters.

The OTR PSF distribution measured experimentally gets wider for longer wavelengths as expected by simulations (see Fig. 5 for comparison).

Table II presents a comparison of the simulated and measured values of the distance between the two lobes extracted from Fig. 12. Both, simulations and experimental data indicate that the size of the PSF reduces when using shorter wavelengths. It shows a reduction by a factor of 1.5 for a wavelength reduction from 600 to $500 \mathrm{~nm}$. Compared to simulations, the measurements show a 10\%-15\% discrepancy, which could be explained by the bandwidth of the color filters used in the experiment, the limited resolution of the camera, and the noise level.

TABLE II. Distance between peaks at the best focus for measurements and simulations and for three wavelengths.

\begin{tabular}{lcc}
\hline \hline Filters & Measurements $(\mu \mathrm{m})$ & Simulations $(\mu \mathrm{m})$ \\
\hline $500 \mathrm{~nm}$ & $6.34 \pm 0.10$ & 5.74 \\
$550 \mathrm{~nm}$ & $7.06 \pm 0.19$ & 7.14 \\
$600 \mathrm{~nm}$ & $9.89 \pm 0.26$ & 8.65 \\
\hline \hline
\end{tabular}



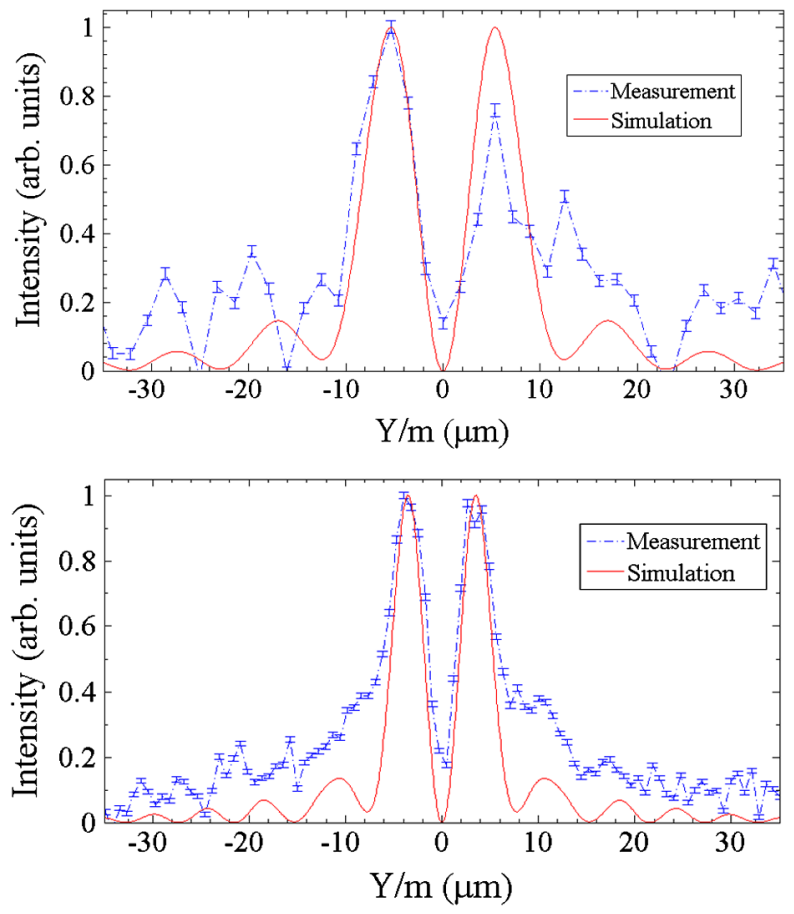

FIG. 13. Vertical projection of the PSF measured and simulated with the plano-convex lens (top) and the achromatic lens (bottom) at the best focus $(\gamma=2500 ; \lambda=550 \mathrm{~nm})$.

\section{Point spread function for different lenses}

Here we present the images obtained experimentally using two different lenses. The first is a $100 \mathrm{~mm}$ focal length plano-convex lens (SLB-30-100-PY2 from SigmaKoki) and the second a $120 \mathrm{~mm}$ focal length achromatic doublet lens (DLB-30-120-PM from Sigma-Koki). They provide optical magnifications of 9.1 and 7.4, respectively.

Figure 13 presents a comparison between the vertical projections measured and simulated for both lenses.

Each lens was tested under similar experimental conditions, in terms of beam charge, size, and energy. The tests were performed using the following procedure: (1) adjusting the nearest quadrupole field strength to get the smallest beam size; (2) adjusting the longitudinal position of the lens to find the image plane; (3) adjusting the vertical height of the lens to avoid misalignment.

As expected, the achromatic lens provides a better correction of chromatic aberrations and thus a PSF that is $34 \%$ narrower than the PSF of the plano-convex lens.

\section{Chromatic aberration}

Two optical band-pass filters, with $25 \mathrm{~nm}$ and $40 \mathrm{~nm}$ bandwidth, have been used in order to study chromatic effects. Figure 14 shows the results obtained with $550 \mathrm{~nm}$ band-pass filters. In this case, the PSF does not change significantly as expected for achromatic lenses. Using the $25 \mathrm{~nm}$ bandwidth optical filter does not seem to affect the shape of the distribution. It only decreases the amount of

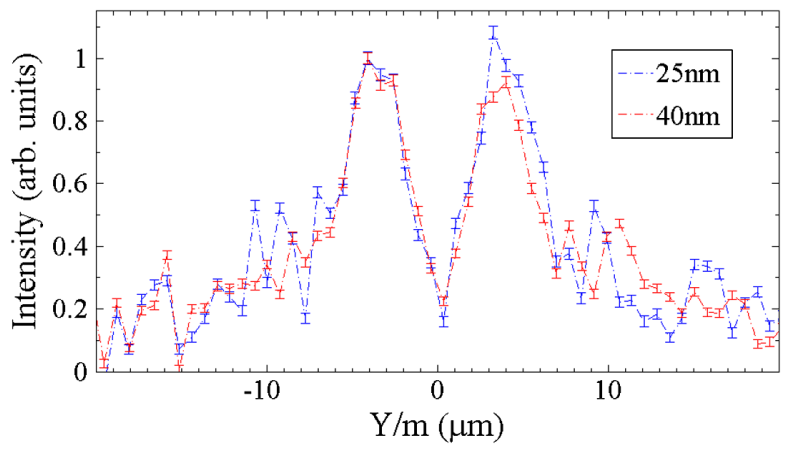

FIG. 14. OTR PSF measured for an achromatic lens with $550 \mathrm{~nm}$ optical filters of 25 and $40 \mathrm{~nm}$ bandwidth.

light intensity available for the measurement deteriorating the signal-to-noise ratio.

ZEMAX simulations can only be performed using a single wavelength. In order to confirm the experimental observations, simulations were performed for three wavelengths, 530, 550, and $570 \mathrm{~nm}$ in order to cover a bandwidth similar to the ones of the optical filters. The position of the lens was set to get the image plane adjusted for the central wavelength of $550 \mathrm{~nm}$ and it was kept constant for the two other wavelengths. No noticeable difference was observed between these simulations, which confirms that chromatic aberrations are kept at a very low level for the achromatic lens and do not limit the actual resolution of the monitor.

\section{E. Effect of diffraction}

Diffraction effects and spherical aberrations were studied both theoretically and experimentally. For that purpose, a motorized iris has been inserted in front of the achromatic lens. Some distributions acquired for different iris diameters are depicted in Fig. 15. The effective diameter of the lens is $30 \mathrm{~mm}$.

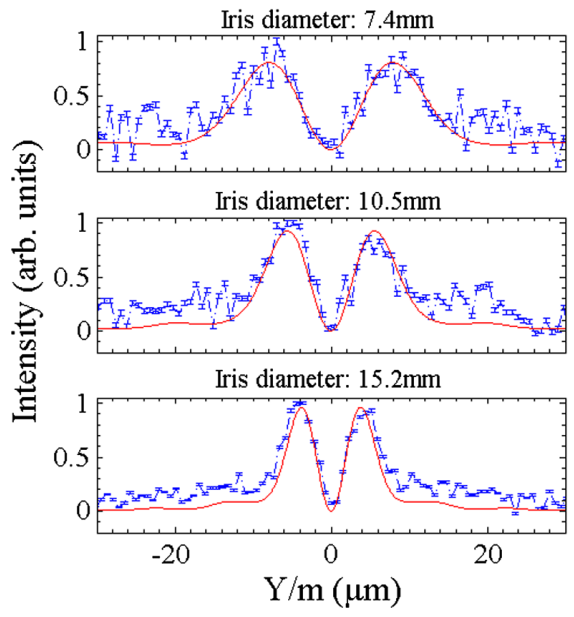

FIG. 15. OTR PSFs measured for different iris diameters $(\gamma=2500 ; \lambda=550 \mathrm{~nm})$. 


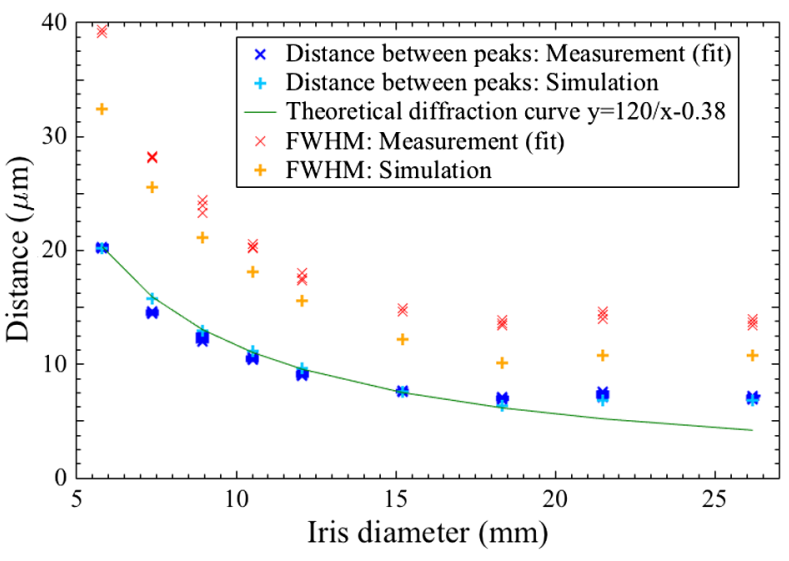

FIG. 16. OTR PSF size for different iris diameters and $\lambda=550 \mathrm{~nm}, \gamma=2500$.

The measured and simulated distances between peaks, as well as the FWHM of the corresponding PSFs, are presented in Fig. 16 as a function of the iris diameter. The size of the PSF increases when decreasing the diameter of the iris. For iris diameters smaller than $18 \mathrm{~mm}$, the optical system is diffraction limited. A theoretical diffraction curve has been added to the plots in Fig. 16 for comparison. For iris diameters larger than $18 \mathrm{~mm}$, the experimental data is above the theoretical diffraction curve, which indicates that the system is dominated by aberrations.

The minimum distance between peaks obtained experimentally was $6.4 \mu \mathrm{m}$ (FWHM OTR PSF $=9.9 \mu \mathrm{m})$ for a wavelength of $550 \mathrm{~nm}$.

\section{CONCLUSION}

We have developed a simulation tool based on the ZEMAX optics simulation software with the aim to optimize the design of the optical transition radiation imaging system and predict their performance. In this paper, we presented simulations of the OTR angular distribution and point-spread function taking into account realistic optical components.

The high-resolution OTR imaging system installed at ATF2 at KEK has been simulated in detail and an excellent agreement between simulations and experimental data has been obtained. Using such a system, vertical beam sizes smaller than $1 \mu \mathrm{m}$ were successfully observed using techniques based on the modulation of the PSF.

For beams with a transverse size similar or slightly larger than the PSF, simulations indicate that the measured beam profile would suffer systematic errors, as large as $100 \%$, that need to be taken into account in the data analysis. This clearly underlines the importance of having a powerful simulation tool for predicting accurately the resolution of OTR imaging systems.

Future study shall investigate the possibility of designing an imaging system working in the UV regime with a telescope made out of elliptical mirrors. This particular arrangement is expected to reduce diffraction, minimize aberrations, and provide a high magnification as required for ultrahigh resolution profile measurements.

Finally, additional studies are currently being carried out to simulate in ZEMAX other types of radiation used for beam diagnostics purposes, like diffraction radiation.

\section{ACKNOWLEDGMENTS}

This work was supported by JSPS KAKENHI Grants No. 23226020 and No. 24654076. We acknowledge the financial support from CERN within CLIC-UK Collaboration.

[1] M. Castellano and V. A. Verzilov, Spatial resolution in optical transition radiation beam diagnostics, Phys. Rev. ST Accel. Beams 1, 062801 (1998).

[2] M. L. Ter-Mikaelyan, High Energy Electromagnetic Processes in Condensed Media (Wiley Interscience, New York, 1972).

[3] V. A. Lebedev, Diffraction-limited resolution of optical transition radiation monitor, Nucl. Instrum. Methods Phys. Res., Sect. A 372, 344 (1996).

[4] M. Castellano, A. Cianchi, G. Orlandi, and V. A. Verzilov, Effects of diffraction and target finite size on coherent transition radiation spectra in bunch length measurements, Nucl. Instrum. Methods Phys. Res., Sect. A 435, 297 (1999).

[5] P. Karataev, Pre-wave zone effect in transition and diffraction radiation: Problems and solutions, Phys. Lett. A 345, 428 (2005).

[6] ZEMAX user's manual, 2013.

[7] P. Karataev, A. Aryshev, S. Boogert, D. Howell, N. Terunuma, and J. Urakawa, First Observation of the Point Spread Function of Optical Transition Radiation, Phys. Rev. Lett. 107, 174801 (2011).

[8] K. Kruchinin et al., Sub-micrometer transverse beam size diagnostics using optical transition radiation, J. Phys. Conf. Ser. 517, 012011 (2014) and references therein.

[9] V. A. Verzilov, Transition radiation in the pre-wave zone, Phys. Lett. A 273, 135 (2000).

[10] T. Aumeyr, M. G. Billing, L. M. Bobb, B. Bolzon, P. Karataev, T. Lefevre, and S. Mazzoni, ZEMAX simulations of transition and diffraction radiation, J. Phys. Conf. Ser. 517, 012026 (2014).

[11] ATF2 Collaboration, ATF2 Proposal, KEK Report No. 2005-2.

[12] G. R. White et al., Experimental Validation of a Novel Compact Focusing Scheme for Future Energy-Frontier Linear Lepton Colliders, Phys. Rev. Lett. 112, 034802 (2014).

[13] L. J. Nevay, S. T. Boogert, P. Karataev, K. Kruchinin, L. Corner, D. F. Howell, R. Walczak, A. Aryshev, J. Urakawa, and N. Terunuma, Laserwire at the Accelerator Test Facility 2 with submicrometer resolution, Phys. Rev. ST Accel. Beams 17, 072802 (2014). 